\title{
Roles of miR-210 in the pathogenesis of pre-eclampsia
}

Jiyun Li, Guimei Wu, Yanmin Cao, Zhi Hou

Third Department of Obstetrical, Hebei Cangzhou Central Hospital, Hebei, China

Submitted: 21 September 2017

Accepted: 20 December 2017

Arch Med Sci 2019; 15, 1: 183-190

DOI: https://doi.org/10.5114/aoms.2018.73129

Copyright (c) 2018 Termedia \& Banach

\section{Abstract}

Introduction: This study aimed to explore the bio-function of miR-210 in the pathogenesis of pre-eclampsia and provide new insights into the diagnosis and treatment of pre-eclampsia.

Material and methods: A JAR cell line cultured in standard or hypoxic conditions was used in this study. Expression levels of miR-210 and PTPN2 were determined using real-time polymerase chain reaction (RT-PCR). Protein and phosphorylation levels were assessed using western blotting. Proliferation of JAR cells was evaluated using MTT assay. Migration and invasion were measured using transwell assay.

Results: Expression of miR-210 increased significantly in a time-dependent manner after hypoxia treatment within $36 \mathrm{~h}(p<0.05)$. miR-210 inhibitor significantly decreased the cell proliferation, migration, and invasion $(p<0.05)$, while miR-210 mimic reversed these findings $(p<0.05)$. Hypoxia significantly suppressed the expression of PTPN2; however, this elevation was abolished by miR-210 inhibitor $(p<0.05)$. Inhibition of PTPN2 or hypoxia significantly increased the proliferation, migration, and invasion of JAR cells, while miR210 inhibitor significantly reversed these changes $(p<0.05)$. The phosphorylation levels of PDGFR, Akt, and Erk were markedly upregulated by hypoxia or si-PTPN2, but this effect was abolished by miR-210 inhibitor $(p<0.05)$.

Conclusions: miR-210 can promote proliferation, migration, and invasion via downregulating PTPN2 in the PDGFR-Akt pathway.

Key words: pre-eclampsia, miR-210, PTPN2, invasion, migration.

\section{Introduction}

Pre-eclampsia (PE) is a leading cause of maternal and morbidity worldwide [1]. This disease, which is characterized by proteinuria and hypertension, usually occurs at about 32 weeks of gestation [2]. It is estimated that approximately $2-8 \%$ of pregnant women suffer from PE [3, 4]. Several risk factors contribute to the pathogenesis of $P E$, including pregnancy age, times, hypertension, anemia, overweight/obesity, urinary tract infection, and hereditary factors $[5,6]$. Despite the progress in the diagnosis and treatments, the current interventions are insufficient to achieve improving effects on the prevention and treatment of $\mathrm{PE}$, as well as its complications [7]. Therefore, it is important to explore the mechanism of PE for further understanding.

A recent study revealed that failure in the vascular remodeling of the maternal spiral arteries is the main pathogenesis of $P E$, which leads to hypoperfusion of the placenta [8]. Specifically, trophoblast invasion is the one of the most important contributors to failure in vascular remodeling [9]. Hunkapiller et al. documented that the Notch signaling pathway plays

\author{
Corresponding author: \\ Dr. Guimei Wu \\ Third Department \\ of Obstetrical \\ Central Hospital \\ 16 Xinhua West Road \\ 061000 Hebei, Cangzhou \\ Hebei, China \\ Phone: +865634856 \\ E-mail: wugmay@126.com
}


a critical role in the endovascular invasion of the trophoblasts [10]. Since micoRNAs (miRNAs) have been demonstrated as therapeutic targets, they could be used as biomarkers in various diseases $[11,12]$. Moreover, they are also reported to play critical roles in the regulation of trophoblast invasion, which participates in the pathogenesis of PE. Li et al. revealed that miR-29b promotes PE via regulating the apoptosis and invasion of trophoblasts [13]. Meanwhile, miR-16 suppresses the proliferation of mesenchymal stem cells in severe PE [14], miR-126 is downregulated in placentas of PE patients, and miR-126 correlates with the decreased expression of vascular endothelial growth factor (VEGF) [15]. Therefore, miRNAs might play critical roles in the regulation of PE pathogenesis.

In order to further explore the potential mechanism of miRNAs in PE, the bio-function of miR-210 and its downstream target were investigated, as well as the potentially involved pathway, so that some useful information could be provided for the diagnosis and treatment of PE.

\section{Material and methods}

\section{Cell culture}

The human placental choriocarcinoma cell line JAR was purchased from American Type Culture Collection (ATCC, Manassas, VA, USA) to explore the functions of trophoblastic cells. JAR cells were maintained in 1640 medium supplemented with $10 \%$ fetal bovine serum, $6 \mathrm{mM}$ glutamine, $20 \mathrm{mM}$ HEPES, $100 \mu \mathrm{g}$ streptomycin and $100 \mathrm{IU}$ penicillin at $37^{\circ} \mathrm{C}$ with a humidified atmosphere of $5 \% \mathrm{CO}_{2}$. For hypoxia treatment, cells were maintained at $37^{\circ} \mathrm{C}$ with a humidified atmosphere of $5 \% \mathrm{CO}_{2}$, $2 \% \mathrm{O}_{2}$ and balanced $\mathrm{N}_{2}$.

\section{Cell transfection}

For knockdown of PTPN2 in JAR cells, short hairpin RNA targeting PTPN2 (sequence: GAAGATGTGAAGTCGTATTAT) or a control (sequence: 5'-AAAGAAATGTCCTACCTTCT-3') was inserted into the plKO.1 vector to construct the shPTPN2 or control plasmid. Then, JAR cells were seeded in a 6-well plate with complete medium, and miR-210 inhibitor (purchased from GE Health Dharmacon, Waltham, MA, USA), miR-210 mimic (purchased from GE Health Dharmacon, USA), si-PTPN2 and si-PTPN2 control were respectively transfected into JAR cells using Lipofectamine 2000 (Invitrogen, USA) following the manufacturer's protocol on the second day. After transfection for more than $48 \mathrm{~h}$, cells were used for the following experiments.

\section{MTT assay}

The JAR cells with different transfections were seeded on a 96-well plate. After culture for
24 and $48 \mathrm{~h}, 200 \mu \mathrm{l}$ of $5 \mathrm{mg} / \mathrm{ml}$ MTT (Sigma, St Louis, MO, USA) was added into each well at $37^{\circ} \mathrm{C}$ for $4 \mathrm{~h}$. Then the medium was discarded and replaced with dimethyl sulfoxide. After shaking for $10 \mathrm{~min}$, the absorbance of each well at $540 \mathrm{~nm}$ was determined using a microtiter plate reader.

\section{Matrigel invasion assay}

The JAR cells $\left(5 \times 10^{5}\right)$ with different expression levels of miR-210 or PTPN2 were plated in 1640 medium without FBS in the upper chamber of a transwell (Corning, Somerset, NJ, USA) coated with Matrigel (BD, MA, USA). Complete growth medium was added to the lower chambers. Then the plate was incubated in standard culture conditions $\left(21 \% \mathrm{O}_{2}\right)$ or hypoxic conditions $\left(2 \% \mathrm{O}_{2}\right)$ at $37^{\circ} \mathrm{C}$ for $72 \mathrm{~h}$. Following this, cells on the upper chamber were removed with a cotton swab, and cells on the underside were stained with hematoxylin (Sigma). For each sample, 5 views were randomly selected and quantified, and the mean value was computed. Each experiment was performed in triplicate, and the mean value was calculated as the final result.

\section{Transwell assay}

Similarly, for the Matrigel assay, $5 \times 10^{5} \mathrm{JAR}$ cells with different expression levels of miR-210 or PTPN2 were separately seeded in 1640 medium without FBS in the upper chamber of a transwell (Corning). Then, the lower chamber was filled with complete growth medium (1640 medium with FBS). Next, the plate was cultured in standard conditions $\left(21 \% \mathrm{O}_{2}\right)$ or hypoxic conditions $\left(2 \% \mathrm{O}_{2}\right)$ at $37^{\circ} \mathrm{C}$ for $72 \mathrm{~h}$. Subsequently, cells on the upper chamber were scraped with a cotton swab, and on the other side were stained with hematoxylin (Sigma). For each sample, 5 views were randomly selected and qualified, and the mean value was obtained as the final result of the sample. Each experiment was performed in triplicate, and the mean value was calculated as the final result.

\section{Quantitative real-time PCR (qRT-PCR)}

After culture or treatment, medium of JAR cells was completely removed, and $1 \mathrm{ml}$ of Trizol agent (Invitrogen) was added to isolate total RNA according to the manufacturer's protocol. After quantification using a NanoDrop spectrophotometer (NanoDrop Technologies, Oxfordshire, UK), $2 \mu \mathrm{g}$ of total RNA was used for mRNA reverse transcription using the high-capacity cDNA Reverse Transcription kit (Applied Biosystems, Inc., Foster City, CA, USA) according to its specific instructions. Meanwhile, $2 \mu \mathrm{g}$ of total RNA was used for miRNA reverse transcription using the TaqMan miRNA Reverse Transcription Kit (Applied Biosystems, 
Inc., Foster City, CA, USA). Then, the expression levels of the gene and miRNA were determined on an ABI PRISM 7900HT PCR system (Applied Biosystems, Foster (ity, CA, USA) with the following system: for the quantification, GAPDH was used to normalize mRNA, and U6 was used to normalize miRNA. Primers of miR-210 and PTPN2 were designed as follows: miR-210-forward, 5'-GTGCAGGGTCCGAGGT-3', and miR-210-reverse: 5'-CTGTGCGTGTGACAGCGGCTGA-3'; U6-forward, 5'-CTCGCTTCGGCAGCACA-3', and U6-reverse, 5'-AACGCTTCACGAATTTGCGT-3'; PTPN2-forward, 5'-TTCCTCTGAACCCCAAACTG-3' and PTPN2-reverse, 5'-GCCTCCAAAAACAAATCCTG-3'; and GAPDH-forward, 5'-CGAGATCCCTCCAAAATCAA-3' and GAPDH-reverse, 5'-TGTGGTCATGAGTCCTTCCA-3'. Then, the fold changes of genes and miRNA were calculated using the $2^{-\Delta \Delta c t}$ method [16]. Each sample was analyzed in triplicate, and the mean value was set as the final expression value.

\section{Western blotting}

Protein lysates from each cell sample were collected, quantified with the Bradford assay, and boiled with loading buffer for $10 \mathrm{~min}$. A total of $10 \mu \mathrm{g}$ of protein was used to run in $10 \%$ or $12 \%$ SDS-PAGE gel, and transferred electrophoretically to a PVDF membrane using a standard method. After blocking with skimmed milk, membrane was incubated with rabbit anti-PTPN2 (1: 1000), rabbit anti-PDGFR (1:2000), p-PDGFR (1:1000), Akt (1 : 3000), p-Akt (1 : 2000), Erk (1 : 3000), p-Erk (1 : 3000), or GAPDH (1: 5000) purchased from Cell Signaling Technology (Denvers, MA, USA), then incubated with secondary rabbit antibodies (1 : 10000, Cell Signaling Technology), and visualized using the enhanced chemical luminescence method.

\section{Statistical analysis}

In the current study, SPSS 20.0 software (IBM, SPSS, Chicago, IL, USA) was used to perform statistical analysis. Continuous variables were presented as mean \pm standard deviation. Comparisons between groups were estimated using Student's $t$-test, and $p<0.05$ was considered statistically significant.

\section{Results}

\section{Hypoxia induces expression of miR-210 in JAR cells}

To investigate the bio-function of miR-210, JAR cells were cultured in standard conditions with $21 \% \mathrm{O}_{2}$ and hypoxic conditions with $2 \% \mathrm{O}_{2}$. The results showed that the mRNA level of miR-210 significantly increased in a time-dependent manner within $48 \mathrm{~h}$ under hypoxic conditions compared

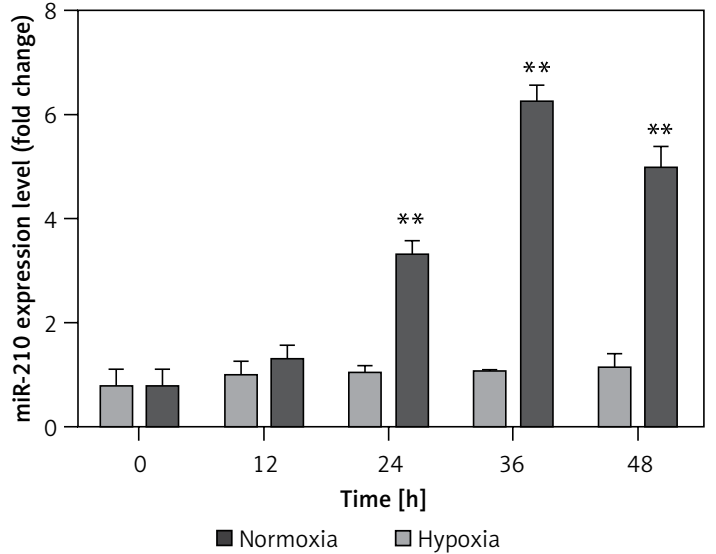

Figure 1. Expression of miR-210 under hypoxia determined using real-time PCR. Compared with the control group, ${ }^{* *} p<0.01$

with the control group (Figure 1), indicating that miR-210 was involved in the response to hypoxia.

miR-210 promotes the cell viability, migration and invasion of JAR cells

For further analysis, miR-210 control, mimic and inhibitor were separately transfected into JAR cells, and the efficacy was determined using RTPCR (Figure $2 \mathrm{~A}$ ). Then, cell viability was measured using MTT, and the result showed that compared with the control group, miR-210 mimic significantly increased the viability of JAR cells, while miR210 inhibitor markedly decreased cell viability of JAR cells (Figure 2 B). Similar tendencies were also identified in the migration and invasion of JAR cells (Figures 2 C, D). These findings indicated that miR-210 could increase cell viability and promote migration and invasion of JAR cells.

\section{PTPN2 is a downstream target of miR-210}

PTPN2 has been reported to be a target of miR210 [17]. In the present study, shRNA was used to inhibit the expression of PTPN2 in JAR cells. Meanwhile, JAR cells were transfected with miR-210 inhibitor or treated with hypoxia to assess the correlation between miR-210 and PTPN2. Then, the expression levels of PTPN2 were measured using RT-PCR and Western blotting. As shown in Figure 3, both si-PTPN2 and hypoxia significantly decreased the expression of PTPN2, while miR-210 inhibitor prevented this elevation. These findings indicated that PTPN2 might be a target of miR-210, and inhibiting the expression of miR-210 could significantly increase the expression of PTPN2.

\section{PTPN2 suppresses the proliferation, migration and invasion of JAR cells}

Effects of PTPN2 on cell proliferation, migration, and invasion were investigated. Both si-PTPN2 
A

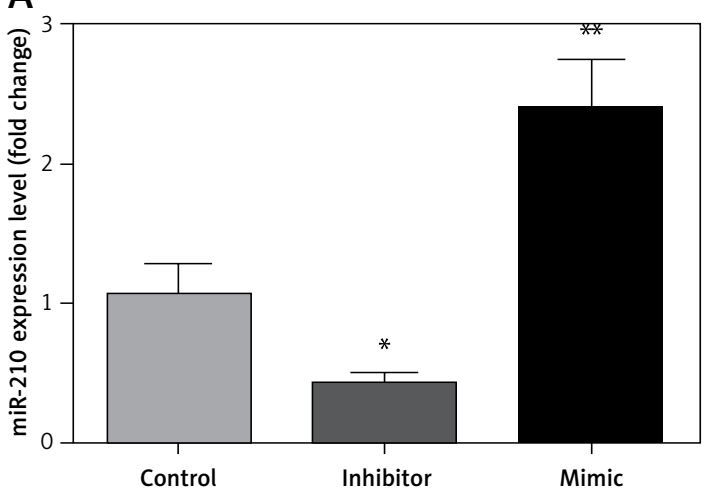

B

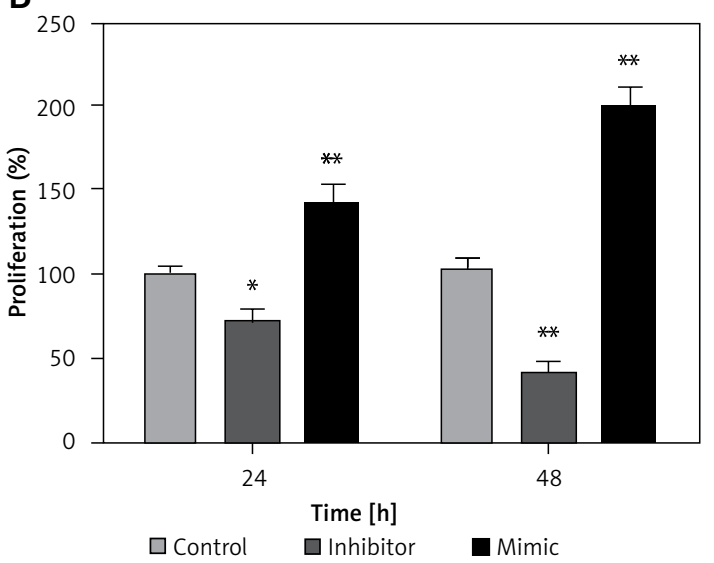

C

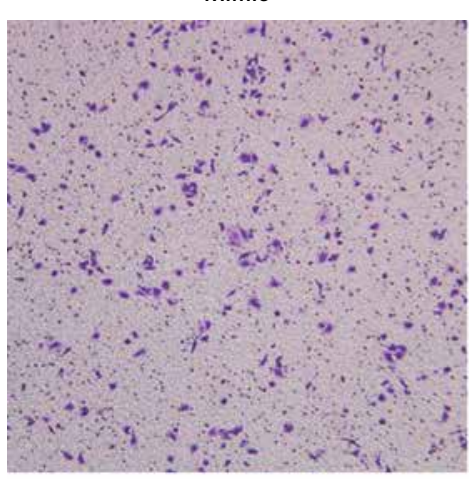

D

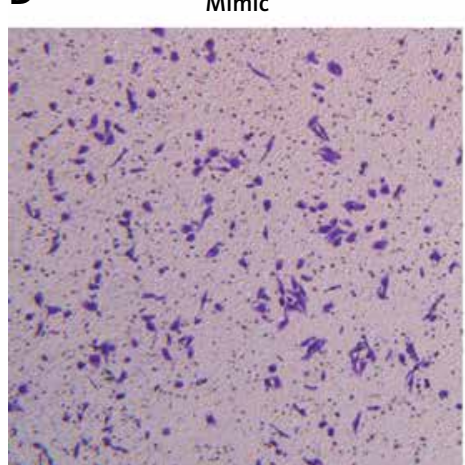

Control

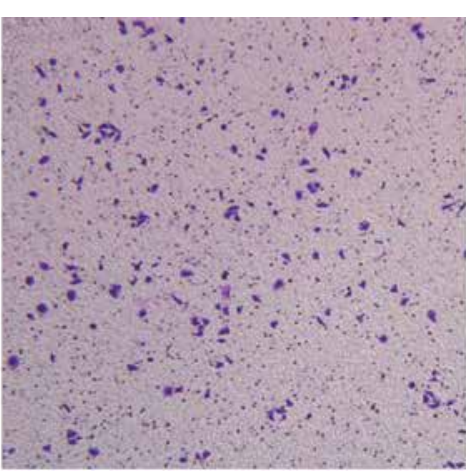

Control

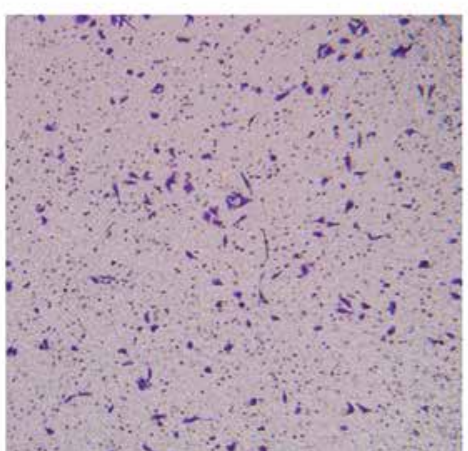

Inhibitor

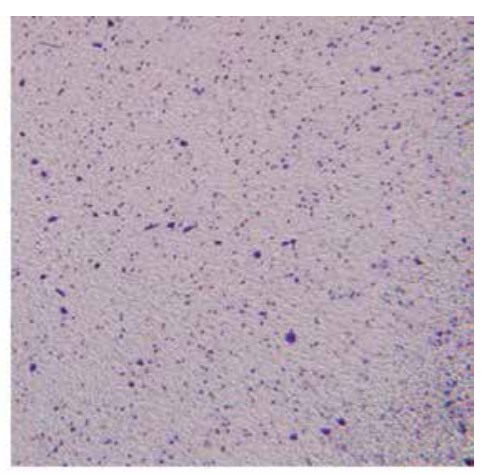

Inhibitor

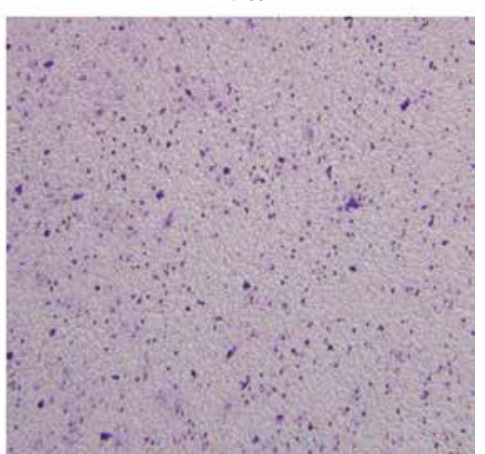

Figure 2. Effect of miR-210 on the proliferation, migration, and invasion of JAR cells. A - Expression of miR-210 determined using real-time PCR, B - cell proliferation determined using MTT, C - cell migration assessed using transwell assay, D - cell invasion estimated using Matrigel assay. Compared with the control group, ${ }^{* *} p<0.01$

and hypoxia significantly increased cell proliferation, and promoted cell migration and invasion of JAR cells, while miR-210 inhibitor reversed these effects of hypoxia and si-PTPN2 (Figure 4).

\section{Pathway of miR-210-PTPN2 involvement}

To explore the potential signaling pathway of miR-210-PTPN2 involved in the current study, the expression and phosphorylation levels of PDGFR, Akt, and ERK were determined by Western blotting (Figure $5 \mathrm{~A}$ ). The quantification of phosphorylation showed that si-PTPN2 and hypoxia treat- ment significantly increased the phosphorylation levels of PDGFR (Figure 5 B), Akt (Figure 5 C), and ERK (Figure $5 \mathrm{D}$ ) compared with the control group, and miR-210 reversed these outcomes, with no significant changes in these levels. These findings indicated that miR-210-PTPN2 might regulate the cell proliferation, migration and invasion via the PDGFR-Akt signaling pathway.

\section{Discussion}

In the current study, the bio-function of miR-210 and its potential target were revealed in JAR cells. 
A

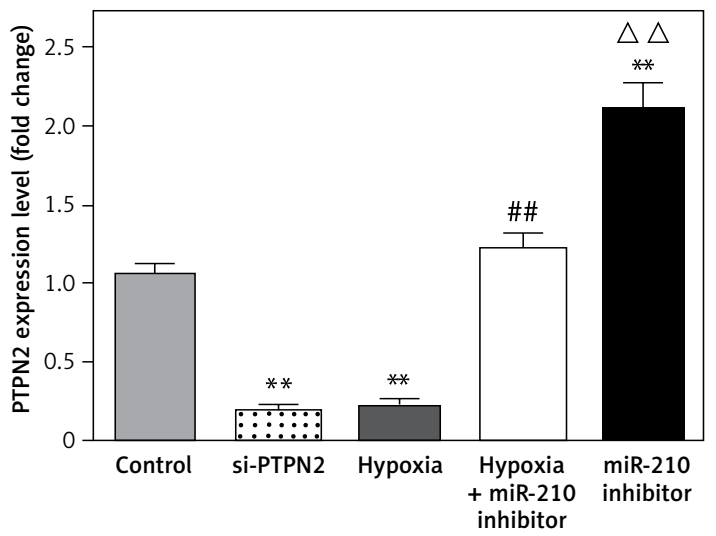

B

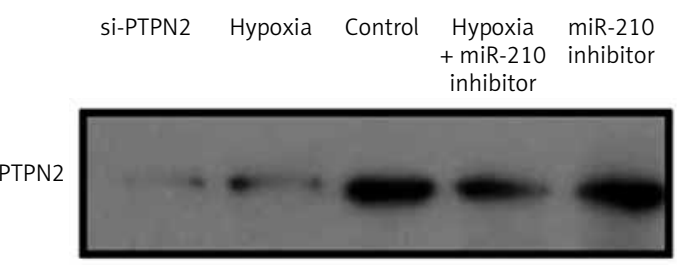

GAPDH

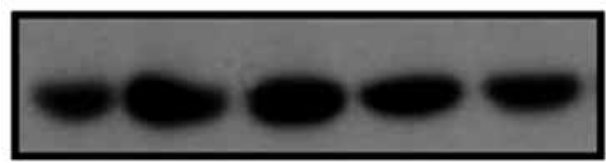

Figure 3. Expression of PTPN2 in cells with different treatment. A - mRNA expression of PTPN2 determined using real-time PCR, B - protein expression of PTPN2 using western blotting

Compared with the control group, ${ }^{* *} p<0.01$; compared with hypoxia + miR-210 inhibitor, ${ }^{* \# p} p<0.01$.

A

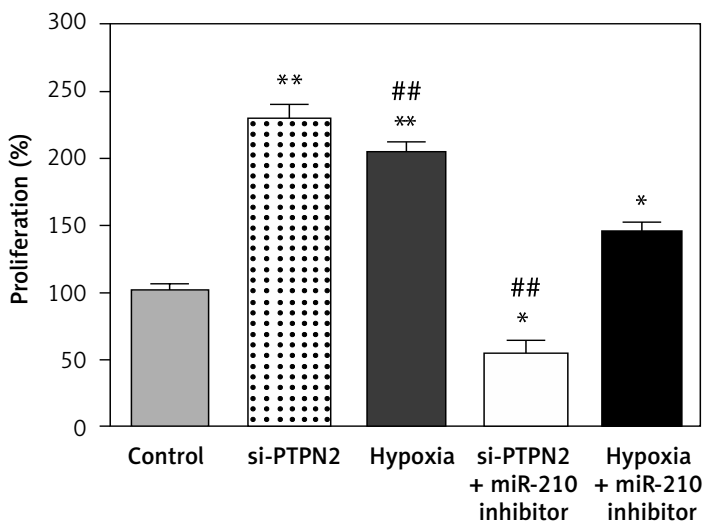

B

Si-PTPN2
+ miR-210 inhibitor

Control
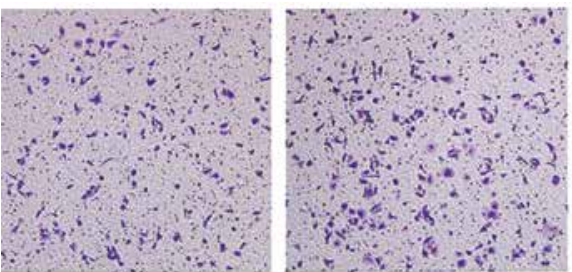

Hypoxia

si-PTPN2

Hypoxia

C

si-PTPN2
miR-210 inhibitor

\section{+ miR-210 inhibitor}
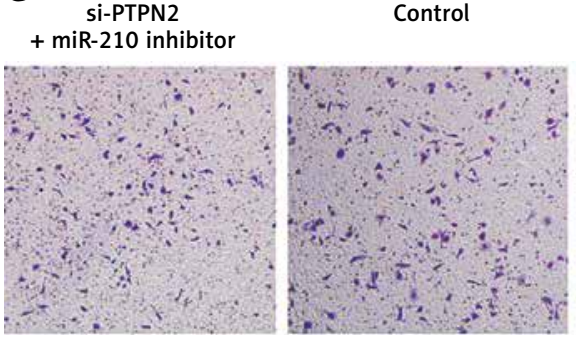

+ miR-210 inhibitor
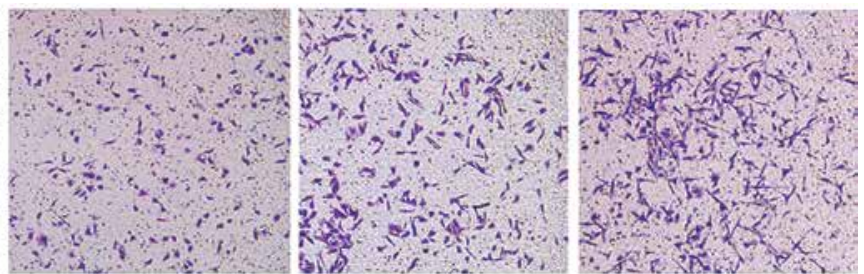

Figure 4. Effect of si-PTPN2 and hypoxia on the proliferation, migration, and invasion of JAR cells. A - cell proliferation determined using MTT, B - cell migration assessed using transwell assay, C - cell invasion estimated using Matrigel assay

Compared with the control group, ${ }^{*} p<0.05$ and ${ }^{* *} p<0.01$; compared with hypoxia + miR-210 inhibitor, ${ }^{\# *} p<0.01$.

After investigation, miR-210 expression increased significantly under hypoxia. Overexpression of miR-210 obviously promoted the cell proliferation, metastasis and invasion of JAR cells, and PTPN2, a target of miR-210, significantly attenuated these elevations via the PDGFR-Akt signaling pathway. 


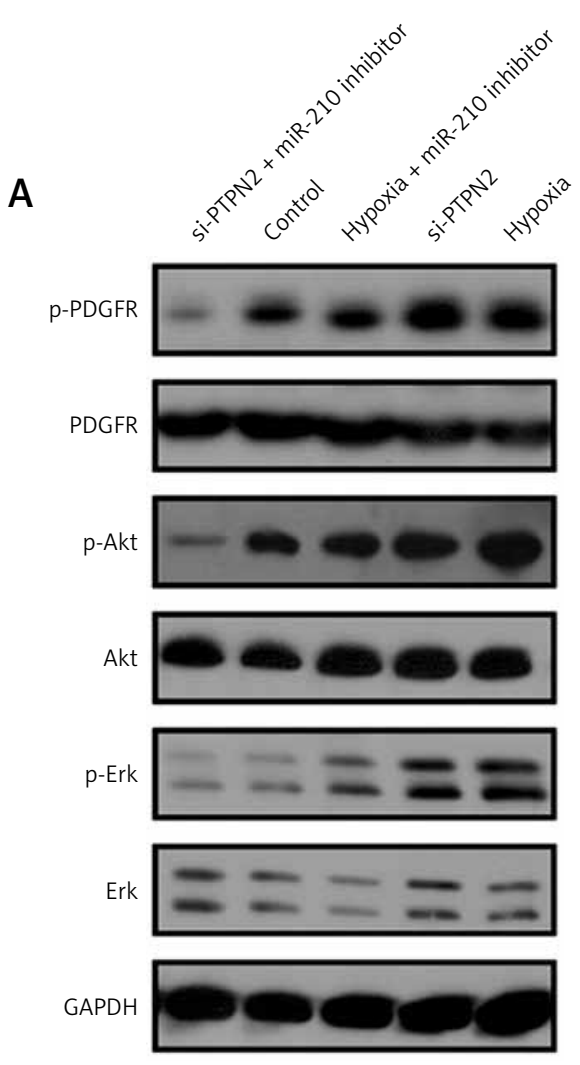

C

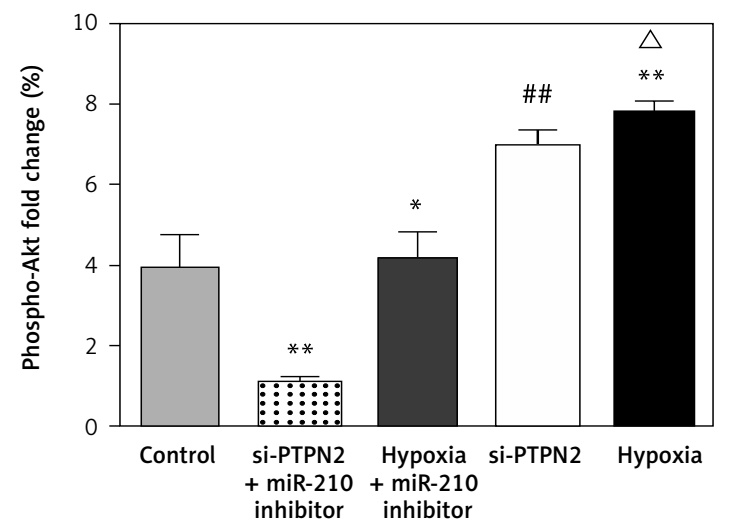

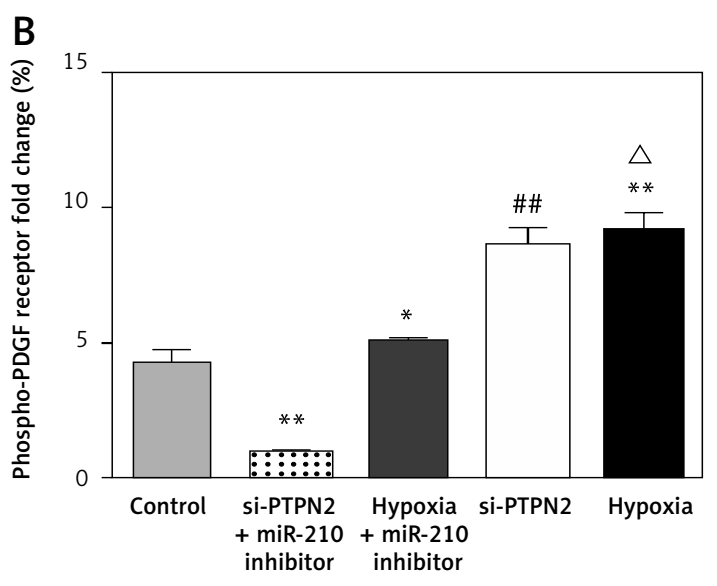

D

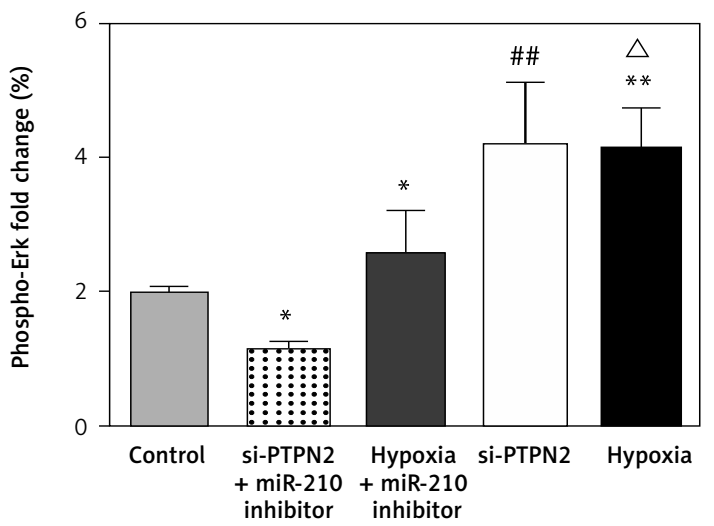

Figure 5. Expression and phosphorylation variations of PDGFR, Akt and Erk. A - Variations determined using western blotting, B - quantification of phosphorylation change of PDGFR, C - quantification of phosphorylation change of Akt, D - quantification of phosphorylation change of Erk

Compared with the control group, ${ }^{*} p<0.05$ and ${ }^{* *} p<0.01$; compared with hypoxia + miR-210 inhibitor, ${ }^{\# \#} p<0.01$.

Among hypoxia-regulated miRNA, miR-210 has been reported to be involved in several diseases. Grosso et al. documented that miR-210 promotes a hypoxic phenotype and radioresistance of human lung cancer cell lines [18]. Meanwhile, Kai et al. reported that miR-210 is also involved in the regulation of hepatocellular carcinoma via HIF- $1 \alpha$ and HIF-3 $\alpha$ [19]. Moreover, McCormick et al. demonstrated that miR-210 is a target of HIF-1 and HIF-2, and is closely correlated with the prognosis of patients with renal cancer [20]. Based on these findings, it can be supposed that miR-
210 plays a critical role in the hypoxia response. Zhang et al. revealed that expression of miR-210 could be up-regulated under hypoxia by NF- $\mathrm{KB}$ transcriptional factor p50 in PE [21]. Both Gan et al. and Nikuei et al. identified that miR-210 and miR-155 might be potential diagnostic markers for $\mathrm{PE}[22,23]$. All these findings imply that miR-210 also might play a crucial role in the pathogenesis of PE. However, the underlying mechanism remains unclear. Complying with the previously published studies, the present study identifies PE with a significant increase in the expression of miR-210 [21, 
$24,25]$. Further analyses showed that the overexpression of miR-210 could significantly promote cell proliferation, migration, and invasion, while miR-210 inhibitor could significantly inhibit these behaviors of JAR cells. These findings indicate that hypoxia induces the elevation of miR-210 and promotes the invasion of trophoblast cells, as well as the failure of arterial remodeling, leading to the occurrence and development of PE.

As an important member of the protein-tyrosine phosphatase (PTP) family, PTPN2 is involved in the regulation of T-cells and its related biological process. Wiede et al. reported that deficiency of PTPN2 enhanced the response of B cells and $T$ cells, as well as systemic inflammation and autoimmunity [26]. Meanwhile, another study reported that PTPN2 could suppress the proliferation of T-cells [27]. Moreover, Spalinger et al. documented that PTPN2 could regulate the differentiation of CD4+ T cells, and inhibit inflammation in the intestine [28]. Recently, Kim et al. demonstrated that miR-210 promotes the proliferation and migration of stem cells derived from adipose tissue via downregulating PTPN2 [29]. Thus, the bio-function of PTPN2 was uncovered in the current study. The analytical results showed that expression of PTPN2 could be decreased by hypoxia, and miR-210 inhibitor could inhibit this downregulation, which was also identified by Adel et al. in PE [30]. Moreover, analyses of cell behaviors showed that si-PTPN2 could induce the proliferation, migration, and invasion of JAR cells, indicating that PTPN2 performed a negative role in the development of PE. However, miR-210 inhibitor could obviously suppress the effect of si-PTPN2 or hypoxia on JAR cells, indicating that PTPN2 might be a downstream target of miR-210 in the pathogenesis of PE. In addition, PDGFR is reported to be common substrate for PTPN2 [31]. Then, the protein and phosphorylation levels of PDGFR were assessed, as well as Akt and Erk, which are involved in the same signaling pathway. The results showed that the phosphorylation levels of PDGFR, Akt, and Erk were significantly increased by hypoxia treatment and si-PTPN2, but this was prevented by miR-210 inhibitor, indicating miR-210-PTPN2 might regulate the development of PE via the PDGFR-Akt signaling pathway. However, the detailed mechanism still needs to be further explored.

Taken together, this study documented that miR-210 plays a critical role in the development of PE via targeting PTPN2, which could inhibit the process of PE via the PDGFR-Akt pathway. Hence, both miR-210 and PTPN2 could serve as potential biomarkers and therapeutic targets of PE. There are some limitations that restrict the power of this study. First, the expression of miR-210 and PTPN2 was not examined in clinical samples due to the small sample size. Second, in vivo validation was not provided in this study owing to deficiency of ethic authorization. Last but not least, the in-depth mechanism of miR-210 in PDGFR-Akt pathway was not well established. Therefore, further exploration is still required to further reveal the mechanism of miR-210 and PTPN2 in PE. Despite these issues, this study provided some basic information on the process of PE. In conclusion, miR-210 expression could be significantly induced under hypoxia, but PTPN2 expression was obviously decreased under hypoxia, and these variations could significantly promote the proliferation, migration, and invasion of JAR cells. Downstream of miR-210, the effects of si-PTPN2 on JAR could be markedly suppressed by miR-210 inhibitor via the PDGFR-Akt signaling pathway. All of this evidence indicates that miR-210 and PTPN2 might act as potential biomarkers and therapeutic targets for the diagnosis and treatment of PE.

\section{Conflict of interest}

The authors declare no conflict of interest.

\section{References}

1. Eastwood KA, Patterson C, Hunter AJ, Mccance DR, Young IS, Holmes VA. Evaluation of the predictive value of placental vascularisation indices derived from 3-dimensional power Doppler whole placental volume scanning for prediction of pre-eclampsia: a systematic review and meta-analysis. Placenta 2017; 51: 89-97.

2. Ahmed R, Dunford J, Mehran R, Robson S, Kunadian V. Pre-eclampsia and future cardiovascular risk among women: a review. J Am Coll Cardiol 2014; 63: 1815-22.

3. Abalos E, Cuesta C, Grosso AL, Chou D, Say L. Global and regional estimates of preeclampsia and eclampsia: a systematic review. Eur J Obstet Gyn Reprod Biol 2013; 170: $1-7$.

4. Duley L. The global impact of pre-eclampsia and eclampsia. Semin Perinatol 2009; 33: 130-7.

5. Olaya-Garay SX, Velasquez-Trujillo PA, Vigil-De Gracia P. Blood pressure in adolescent patients with pre-eclampsia and eclampsia. Int J Gynaecol Obstet 2017; 138: 335-9.

6. Liu S, Joseph KS, Liston RM, et al. Incidence, risk factors, and associated complications of eclampsia. Obstet Gynecol 2011; 118: 987-94.

7. Roberge S, Sibai B, Mccaw-Binns A, Bujold E. Low-dose aspirin in early gestation for prevention of preeclampsia and small-for-gestational-age neonates: meta-analysis of large randomized trials. Am J Perinat 2016; 33: 781-5.

8. Powe CE, Levine RJ, Karumanchi SA. Preeclampsia, a disease of the maternal endothelium: the role of anti-angiogenic factors and implications for later cardiovascular disease. Circulation 2011; 123: 2856-69.

9. Chaiworapongsa T, Chaemsaithong P, Yeo L, Romero R. Pre-eclampsia part 1: current understanding of its pathophysiology. Nat Rev Nephrol 2014; 10: 466-80.

10. Hunkapiller NM, Gasperowicz M, Kapidzic M, et al. A role for Notch signaling in trophoblast endovascular invasion and in the pathogenesis of pre-eclampsia. Development 2011; 138: 2987-98. 
11. Momtazi AA, Derosa G, Maffioli P, Banach M, Sahebkar A Role of microRNAs in the therapeutic effects of curcumin in non-cancer diseases. Mol Diagn Ther 2016; 20: 335-45.

12. Zhao JJ, Chen PJ, Duan RQ, et al. miR-630 functions as a tumor oncogene in renal cell carcinoma. Arch Med Sci 2016; 12: 473-8.

13. Li P, Guo W, Du L, et al. MicroRNA-29b contributes to pre-eclampsia through its effects on apoptosis, invasion and angiogenesis of trophoblast cells. Clin Sci 2013; 124: 27-40.

14. Wang Y, Fan H, Zhao G, et al. miR-16 inhibits the proliferation and angiogenesis-regulating potential of mesenchymal stem cells in severe pre-eclampsia. FEBS J 2012; 279: 4510-24.

15. Hong F, Li Y, Xu Y. Decreased placental miR-126 expres sion and vascular endothelial growth factor levels in patients with pre-eclampsia. J Int Med Res 2014; 42: 1243-51.

16. Livak KJ, Schmittgen TD. Analysis of relative gene expression data using real-time quantitative PCR and the $2-\Delta \Delta C T$ method. Methods 2001; 25: 402-8.

17. Kim J, Park S, Song S, Kim J, Sung J. Reactive oxygen species-responsive miR-210 regulates proliferation and migration of adipose-derived stem cells via PTPN2. Cell Death Dis 2013; 4: e588.

18. Grosso S, Doyen J, Parks SK, et al. MiR-210 promotes a hypoxic phenotype and increases radioresistance in human lung cancer cell lines. Cell Death Dis 2013; 4 e544.

19. Kai AK, Chan LK, Lo RC, et al. Downregulation of TIMP2 via HIF-1alpha/miR-210/HIF-3 $\alpha$ regulatory feedback circuit enhances cancer metastasis in hepatocellular carcinoma. Hepatology 2016; 64: 473-87.

20. Mccormick RI, Blick C, Ragoussis J, et al. miR-210 is a target of hypoxia-inducible factors 1 and 2 in renal cancer, regulates ISCU and correlates with good prognosis. Br J Cancer 2013; 108: 1133-42.

21. Zhang Y, Fei M, Xue G, et al. Elevated levels of hypoxia-inducible microRNA-210 in pre-eclampsia: new insights into molecular mechanisms for the disease. J Cell Biochem 2012; 16: 249-59.

22. Gan L, Liu Z, Wei M, et al. MiR-210 and miR-155 as potential diagnostic markers for pre-eclampsia pregnancies. Medicine 2017; 96: e7515.

23. Nikuei P, Davoodian N, Tahamtan I, Keshtkar AA. Predictive value of miR-210 as a novel biomarker for pre-eclampsia: a systematic review protocol. BMJ Open 2016; 6: E011920.

24. Wang $H$, Flach $H$, Onizawa $M$, Wei L, Mcmanus MT, Weiss A. Negative regulation of HIF1a expression and Th17 differentiation by the hypoxia-regulated microRNA miR-210. Nat Immunol 2014; 15: 393-401.

25. Dang K, Myers KA. The role of hypoxia-induced miR-210 in cancer progression. Int J Mol Sci 2015; 16: 6353-72.

26. Wiede F, Sacirbegovic F, Leong YA, Yu D, Tiganis T. PTPN2-deficiency exacerbates $T$ follicular helper cell and $B$ cell responses and promotes the development of autoimmunity. J Autoimmun 2017; 76: 85-100.

27. Wiede F, La Gruta NL, Tiganis T. PTPN2 attenuates T-cel lymphopenia-induced proliferation. Nat Commun 2014; 5: 3073 .

28. Spalinger MR, Kasper S, Chassard C, et al. PTPN2 controls differentiation of $\mathrm{CD}^{+}{ }^{+} \mathrm{T}$ cells and limits intestinal inflammation and intestinal dysbiosis. Mucosal Immunol 2015; 8: 918-29.
29. Kim JH, Park SG, Song SY, Kim JK, Sung JH. Reactive oxy gen species-responsive miR-210 regulates proliferation and migration of adipose-derived stem cells via PTPN2. Cell Death Dis 2013; 4: e588.

30. Adel S, Mansour A, Louka M, Matboli M, Elmekkawi SF, Swelam N. Evaluation of microRNA-210 and protein tyrosine phosphatase, non receptor type 2 in pre-eclampsia. Gene 2016; 596: 105-9.

31. Stgermain JR, Taylor P, Zhang W, et al. Differential regulation of FGFR3 by PTPN1 and PTPN2. Proteomics 2015; 15: 419-33. 